\section{Ulrike Schwab}

\section{Erzähltext und Spielfilm}

Zur Ästhetik und Analyse der Filmadaption

Berlin: Lit, 2006. - 388 S.

(Reihe Geschichte, Zukunft, Kommunikation; 4)

ISBN 3-8258-9057-0

(Zugl.: Halle: Univ., Habil.-Schrift, 2004)

Das Resümee dieser voluminösen Studie beginnt mit dem Satz: „Die vorliegende Arbeit nahm ihren Weg über diverse Positionen des wissenschaftlichen Diskurses." Es folgt eine Aufzählung, die nicht alle Themen, die angesprochen werden, nennt, die aber gleichwohl anzeigt, dass das thematische Spektrum, das die Verfasserin verfolgt hat, äußerst umfangreich ist. Die Studie, so heißt es weiter, verhandelt „die kommunikationstheoretische These, dass sich Information beim Transfer (material) transformiert und je kompatible Kommunikationssysteme bedingt“. In diesen Zusammenhang gehört der mediengeschichtliche Aspekt, dass es sich bei „Erzähltext" und „Spielfilm“ um „zwei heterogen strukturierte Informationssysteme" handelt. Hier greift die Verfasserin auf Gieseckes grundlegende Studie zur Einführung des Buchdrucks zurück.

Es wäre durchaus von Interesse, Erzähltexte und Spielfilme mit dem Ansatz von Michael Giesecke zu überprüfen und die Wege und auch Grenzen aufzuzeigen, die dieser Ansatz absteckt, wenn es um Kinematographie und Buch-Literatur geht. Mit einer solchen Fragestellung könnte man einige grundlegende Veränderungen benennen, die zwischen dem Wechsel von der Manuskript-Kultur zur Gutenberg-Galaxis und der Einführung des Kinos in einer Zeit sich ausdifferenzierender Massenmedien stattfinden. Die Verfasserin geht diesen Weg indes nicht, sie übernimmt Kategorien von Giesecke, ohne die Relationen und Differenzen zu reflektieren oder zu problematisieren.

In ihrer Liste von Diskursen, die sie im Verlauf ihrer Arbeit angesprochen hat, folgen als nächste die „rezeptionspsychologische Forschungssicht" und "die Kontroverse zum Wert individuell erschaffener und industriell gefertigter Kunst“. Der letzte Aspekt, der das viel verhandelte Thema der Reproduzierbarkeit anspielt, wird zwar - anfangs mit Verweis auf Walter Benjamin und Erwin Panofsky - immer wieder erwähnt, aber eine theoretische Fundierung dieser Problematik bleibt aus. Das ist kein Zufall. Die Arbeit vermeidet Theoriedebatten absichtlich. Ihr Generalvorwurf an die bisherigen Forschungen zur Adaptionsproblematik, die sie insgesamt stark simplifizierend referiert, lautet, dass in diesen Forschungen „die theoretische Herleitung alles, der konkrete Befund fast nichts" bedeute. Eine solche Einschätzung übersieht-und das ist ein Mangel dieserStudie -, dass Aussagen über Spielfilme ebenso wie über Filme nach literarischen Vorlagen ohne theoretische Fundierung auf einer rein deskriptiven Ebene verbleiben. Das mag man gerade noch akzeptieren, wenn es um konkrete Fallbeispiele geht, wenn Materialien zusammengetragen und ausgewertet werden, aber das führt nicht weit, wenn man sich einem theoretisch so komplexen Problem nähert, wie es das Thema der Adaption nun einmal ist. Das wird z. B. deutlich, wenn die Verfasserin aus Anleitungen zum Drehbuchschreiben Regeln für die „dramaturgische Bearbeitung des Erzählstoffes“ ableitet. Die ganz unterschiedlichen Diskurszusammenhänge solcher Manuals und der wissenschaftlicher Arbeiten werden nicht reflektiert. Hinzu kommt, dass die Verfasserin aus solchen Manuals Generalisierungen ableitet, die für das breite und heterogene Spektrum an Adaptionen, das es mittlerweile gibt, nichtssagend werden. Das verweist auf ein zweites grundlegendes Problem, vor das diese Publikation den Leser stellt. Es geht weder um eine theoretische noch um eine historische Fragestellung. Es geht auch nicht um eine Systematik der Probleme, die mit dem gestellten Thema einhergehen, wie die Heterogenität der Diskurse, die angesprochen werden, zeigt.

Die Verfasserin analysiert im Anschluss an ihren Durchgang durch die verschiedenen Diskurse drei Filmadaptionen - „The Fallen Idol“ und zwei Filme nach Theodor Fontanes Roman „Effi Briest“. Hier werden, insgesamt durchaus konventionell, aber analytisch genauer als in den vorangegangenen Kapiteln, die Vorlagen und ihre Filme analysiert. Etwas breit geraten sind Ausführungen zu Fontane und seinem Roman, die aus der Fontane-Forschung hinlänglich bekannt sind. Das gleiche gilt für die Kontextualisierung der beiden Effi-Filme. Hier muss der Leser manches lesen, was zwar richtig ist, aber in der Sache in diesem Zusammenhang wenig differenziert bleiben muss.

Das Vorhaben, Grundlegendes über Adaptionen zu sagen, ohne theoretisch zu argumen- 
tieren und ohne ein Abstraktionsniveau anzustreben, führt zu vielen begrifflichen und argumentativen Ungenauigkeiten. Ein Beispiel: Für den Erzähltext, so legt die Autorin fest, hat der Satz als „der kleinste funktionale Baustein“ $\mathrm{zu}$ gelten, für den Spielfilm wird „die Einstellung als kleinster funktionaler Baustein angenommen". Mit solchen Festlegungen, die nicht weiter diskutiert werden, schiebt die Verfasserin eine in Filmtheorien lange verhandelte Problematik einfach beiseite. Die „Taxonomie“ für die „systematische Beschreibung von fiktionalen Charakteren " führt zu einer Merkmalsliste, die stark vereinfacht, indem Charaktere z. B. als "naturalistisch“, ,stilisiert“, „kohärent“, ,ganzheitlich“ oder "komplex“ bestimmt werden. Wenn „Kameraführung, Raumdesign, Montage, Ton-/Musikbeitrag und Schauspielen" als „fünf Gestaltungssektoren der (Audio-)Visualisierung" bestimmt werden und festgestellt wird, dass jeder „Einzelbereich“ eine „eigene materiale Grundlage, eine eigene Realisierung und eigene Formprinzipien" hat, so wäre es angezeigt, auf die Interdependenzen und Relationen, die hier gleichermaßen zu beobachten sind, zu verweisen.

Insgesamt fällt auf, dass die Studie einige wichtige medienwissenschaftliche und auch filmwissenschaftliche Debatten der letzten Jahrzehnte nicht aufgreift. So bezieht sie sich zwar auf die einschlägigen filmwissenschaftlichen Studien, z. B. von Biodwell und Thompson, aber weder die Diskussionen, die diese Studien initiiert haben, werden herangezogen noch die Aporien, die mit diesem Ansatz gegeben sind, erwähnt. Der Bezug zu Chatman ist zwar einerseits von großer Bedeutung, bleibt andererseits aber in der Argumentation eher peripher. Ein anderer Aspekt kommt hinzu: Es heißt zwar an einer Stelle, das Drehbuch sei eine „intermediale Textart“, aber die gesamte Diskussion, inwiefern die Adaptionsforschungen im Kontext intermedialer Fragestellungen situiert und differenziert werden können, bleibt hinter dem Horizont dieser Studie.

Die Autorin erhebt am Ende den Anspruch, mit ihrem „modellhafte(n) Aufriss von der Ensembletätigkeit der Erzählinstanzen und Parameter" und der Konkretisierung durch „Sachwissen zur Produktion“ einen „Arbeitsstand“ präsentiert zu haben, von dem aus „ein anderer Weg der Adaptionsforschung nicht mehr denkbar" erscheint. Wer so ambitioniert präsentiert, muss Erwartungen vergleichbarer Art erfüllen. Irmela Schneider 